\title{
Numerical Analysis of Electron Optical System with Microchannel Plate
}

\author{
Alla Shymanska
}

Received: date / Accepted: date

\begin{abstract}
This paper describes a numerical development of image converters and intensifiers which incorporate an inverting electron optical system (EOS) and a microchannel plate (MCP) as an amplifier. The numerical design of the system includes calculation of the electrostatic field in the device, trajectories of electrons emitted from a photocathode, and determination of the modulation-transfer-function (MTF) which gives the objective estimation for the image quality.

Results of the numerical experiments are shown, and the EOS with optimized characteristics is developed. It provides the nearly flat image surface, determines the position of the surface of the best focus, minimizes the image distortion and reduces a noise factor of the MCP.
\end{abstract}

Keywords Electron optical system - Microchannel plate - Electrostatic field · Electron trajectories . Modulation-transfer-function · Numerical analysis

\section{Introduction}

Within its physical limitations the human eye is an efficient and flexible photon detector which provides the observer with information about the surrounding world. Physical limitations include wavelength limitation and sensitivity limitation [15]. These limitations can be improved by using image converters and intensifiers. Image converters give access to a larger spectral domain of electromagnetic radiation than the human eye, and image intensifiers amplify low light images and increase

\section{A. Shymanska}

School of Computing and Mathematical Sciences, Auckland University of Technology, Private Bag 92006, Auckland 1142,

New Zealand.

E-mail: alla.shymanska@aut.ac.nz the eye sensitivity. Gains of up to $10^{6}$ in image converters and intensifiers are achieved in a single tube by placing a microchannel plate (MCP) close to a phosphor screen [4], [8], [18], [19], [22]. Such devices have found wide applications in different areas of science, engineering, medicine etc.

The process of image conversion and intensification in systems with rotational symmetry is as follows: (a) electromagnetic radiation from a certain part of the spectrum is absorbed by a photocathode; (b) an optical image is converted into an electron image by means of photoemission; (c) the photoelectrons of this electron image are accelerated and focused by an electron optical system (EOS), and intensified by the MCP; (d) the intensified electron image is converted into a visible image by a luminescent process on a screen. Figure 1 shows a cross section of such a device and its important elements: a photocathode, an electron-optical system, MCP and a luminescent screen.

A microchannel amplifier is a thin plate made up of a large number of single channels, each of which acts as an electron multiplier [16], [22]. For each primary electron entering a channel, a large pulse of electrons will exit as a result of multiple collisions of both primary and secondary electrons with the inner secondaryemission layer as they traverse the length of the channel. It is a compact, efficient amplifier of two-dimensional electron-images and provides several advantages such as very high gain, good resolution and small size.

However, the inverter tube with MCP has one major design restraint on the electron lens: the image plane must be flat to match the microchannel plate. Also, to provide the best image quality, the plate position should coincide with the surface of the best focus. Parameters of the system should be chosen to minimize a noise factor of the MCP [16] which is most important at 
low levels of illuminance. The resolution and the noise factor of the system affect its major parameter, the visual acuity of an imaging device [14]. Moreover, in the inverter tube the EOS transfers the image from photocathode to the plate with some distortion which should be minimized.

The fundamental problem in an electron-optical system is to devise an arrangement of electrodes and applied potentials which will cause electrons to travel along predetermined paths to focus in a point or plane. There are different techniques for designing of charged particle optical system [9], [10], [11], [13] which can be split into two major groups: analysis and synthesis. The analysis of an electrostatic field distribution and trajectories of electrons for given geometry and fixed potentials of the electrons is used here for the investigation of an inverter tube with MCP. The analysis includes numerical calculation of the field distribution, trajectories of electrons emitted from a photocathode, and determination of the modulation-transfer-function (MTF). The modulation transfer function defines a visual acuity of the image intensification devices [14], and the resolving power of the device can be improved by improving its modulation transfer function. It is especially important for the devices which are designed for conditions of low illumination, for example, for the night vision.

Section 2 describes a numerical algorithm for calculating a potential distribution inside systems with rotational symmetry and complicated boundaries.

Section 3 provides an algorithm for finding the electron trajectories.

Section 4 describes numerical experiments which have been conducted to optimize the electron optical system in terms of the image quality.

Section 5 describes the algorithm for calculating the modulation transfer function of EOS with rotational symmetry, and the MTF of the channel amplifier.

Section 6 provides some results of numerical calculations of MTFs. The MTFs of the EOS, the MTF of microchannel amplifier, and the total MTF of the imagine system are calculated.

\section{Determination of the Potential Field.}

To analyze the potential distribution inside EOS, the essential first stage is to determine the position of the equipotential surfaces in the space under investigation. Due to the rotational symmetry of the system (Fig.1) the electrostatic field has been calculated here in half of the cross-section of the device.

Formally this is a matter of finding a solution to the Laplace's partial differential equation expressed in cylindrical coordinates as follows:

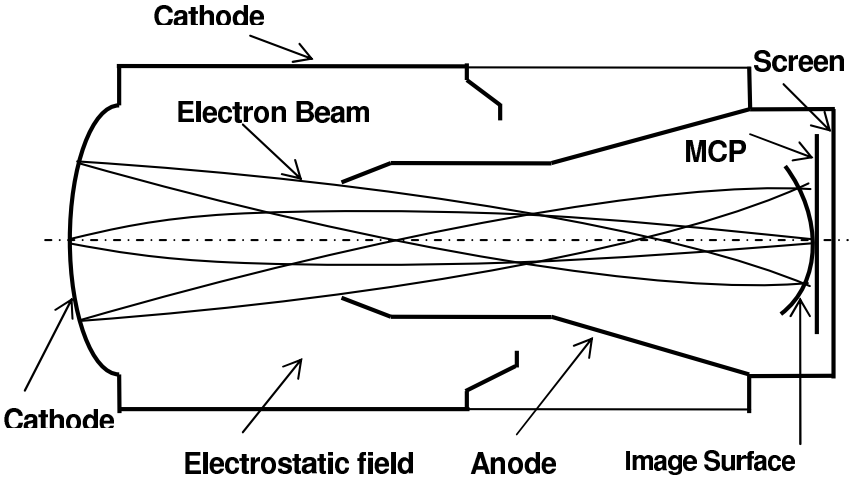

Fig. 1 Electron - optical system

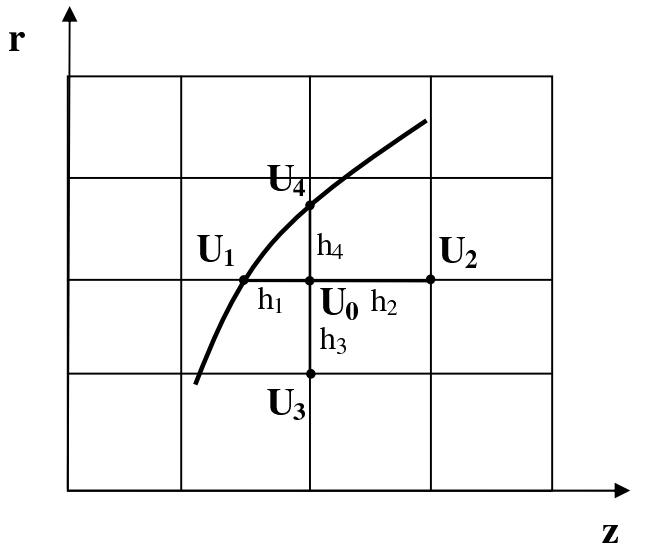

Fig. 2 Grid and finite difference star

$\frac{\partial^{2} U}{\partial z^{2}}+\frac{1}{r} \frac{\partial U}{\partial r}+\frac{\partial^{2} U}{\partial r^{2}}=0$

where $U(z, r)$ is the potential at any point $(z, r), z$ is the coordinate along the axis of symmetry and $r$ is the off-axis radius.

This problem relates to the classical mixed problem for the equation of Laplace in some region with Dirichlet and Neumann boundary conditions. The function $U=f(z, r)$ is defined on the most of the boundaries, and the homogeneous Neumann boundary conditions are imposed on the symmetry axis [10], [11].

The finite difference method is used to obtain the field distribution [13]. The simulation domain is discretised using a regular grid (Fig.2).

The resulting set of finite difference equations is obtained using the Taylor's expansion about the centre node $U_{0}$ with $z=z_{0}$ and $r=r_{0}$. The partial derivatives in (1) are replaced by corresponding difference approximations which are expressed in terms of local potential values. 
For complicated and curved boundaries the Laplace's difference equation is obtained in terms of four different steps $h$ from the central node:

$$
\begin{aligned}
& \frac{2}{h_{1}\left(h_{1}+h_{2}\right)} U_{1}+\frac{2}{h_{2}\left(h_{1}+h_{2}\right)} U_{2}+\frac{2 r_{0}-h_{4}}{r_{0} h_{3}\left(h_{3}+h_{4}\right)} U_{3}+ \\
& +\frac{2 r_{0}+h_{3}}{r_{0} h_{4}\left(h_{3}+h_{4}\right)} U_{4}-\left[\frac{2}{h_{1} h_{2}}+\frac{2 r_{0}-h_{4}+h_{3}}{r_{0} h_{3} h_{4}}\right] U_{0}=0 .
\end{aligned}
$$

For the interior equidistant points, where $h_{k} \equiv h$, the difference equation is:

$\frac{1}{h^{2}}\left[U_{1}+U_{2}+\left(1-\frac{h}{2 r_{0}}\right) U_{3}+\left(1+\frac{h}{2 r_{0}}\right) U_{4}\right]-4 U_{0}=0$.

On the axis of symmetry $\frac{\partial U}{\partial r}=0$ and Laplace's difference equation can be written in the following form:

$\frac{1}{h^{2}}\left(U_{1}+U_{2}+4 U_{4}-6 U_{0}\right)=0$.

Therefore, one of the Laplacian computational formulae (2), (3) or (4) is applied at each of the interior mesh points, and the result is the system of simultaneous linear algebraic equations.

The solution of this system of simultaneous equations is obtained by the iterative successive overrelaxation (SOR) method [23]. For this method the Laplacian difference equation is rewritten in the following form:

$U_{i j}=U_{i j}+\omega r_{i j}$

where $r_{i j}$ is the residual term, and $\omega$ is the relaxation parameter which lies in the range $1 \leq \omega \leq 2$. It has been found that for the system described here the optimal value of $\omega$ is 1.99 .

Figure 3 shows the potential distribution (given by the equipotential lines) in the cross-section of the EOS shown in 1 with the following dimensions: the cathode radius $=0.8$, the length of the system $=2.6$, the cathode potential $=0$, and the anode potential $=1$. All dimensions here and further are normalized by the radius of the cathode cylinder.

As shown in [5], [6], and [17] an electrostatic lens, formed by MCP-screen field penetration into channels, at the output of a channel has a significant influence on the electron trajectories and the spatial resolution (quality of the image). The potential distribution in the field of the lens depends on the field intensity in the MCP-screen gap, the channel diameter, and the sputtering depth of the contact layer at the channel output ("end spoiling" [6]).

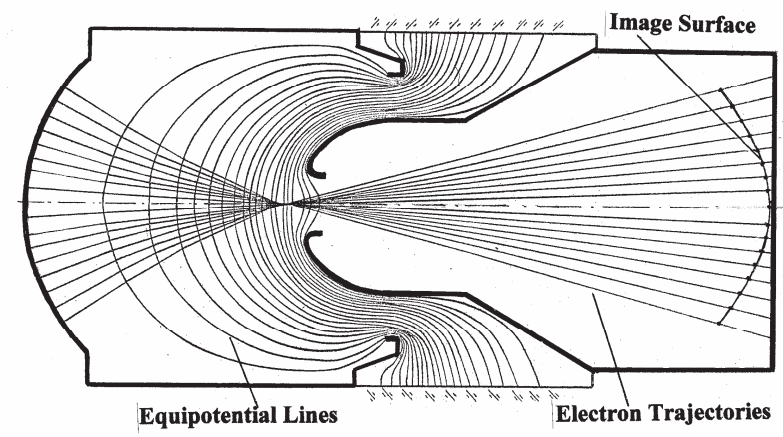

Fig. 3 Equipotential lines and central meridional trajectories of electron beams

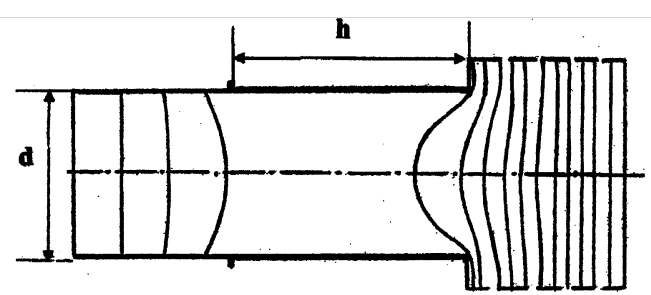

Fig. 4 Electrostatic field at the exit of the channel

Figure 4 shows the electrostatic lens at the exit of a single channel (the fringe field), calculated here using the algorithm described above. Computational results were obtained for the channel diameter $d=10 \mu \mathrm{m}$, the voltage on the channel $U_{c}=800 \mathrm{~V}$, the strength of the electrostatic field between MCP and the screen $E=5$ $\mathrm{kV} / \mathrm{mm}$, and the sputtering depth of the contact layer at the channel output $h=1.5 d$.

\section{Motion of Electrons in the Potential Field}

The equation of the motion of charged particles through the electric field is the Lorentz force:

$\mathbf{F}=q \mathbf{E}$,

where $\mathbf{F}$ is the force on the charged particle, $q$ is the charge, $\mathbf{E}$ is the electric field. In the cylindrical coordinate system, equations of motion of electrons can be written as:

$$
\begin{aligned}
& \frac{d^{2} z}{d t^{2}}=\frac{e}{m} E_{z}, \\
& \frac{d^{2} r}{d t^{2}}=\frac{e}{m} E_{r}+\frac{r_{0}^{2} V_{\varphi_{0}}^{2}}{r^{3}}, \\
& \frac{d \varphi}{d t}=\frac{r_{0}}{r^{2}} V_{\varphi_{0}}
\end{aligned}
$$




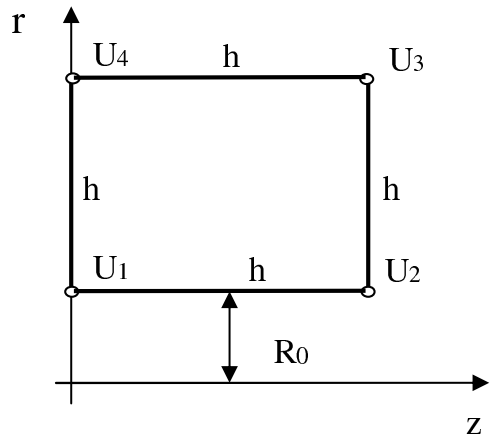

Fig. 5 The grid for calculating the strength of the electrostatic field in the channel

where $t$ is time, $E_{r}=\frac{\partial U}{\partial r}$ and $E_{z}=\frac{\partial U}{\partial z}$ are the radial and axial components of the strength of the electrostatic field respectively, $U=U(z, r)$ is the potential distribution, $r_{0}$ is the initial electron coordinate, $V_{\varphi_{0}}$ is the initial azimuthal component of the electron velocity, $e$ and $m$ are electron charge and mass respectively.

The system of equations (7) is solved by the use of the Runge-Kutta method.

The strength of the electrostatic field at the exit of a single channel and inside the EOS is calculated using different interpolating polynomials.

a). For the fringe field at the exit of a channel the harmonic polynomial via potentials at four mesh points (Fig.5) have been used [7]:

$U(\varsigma, r)=a_{00}+a_{10} \varsigma+a_{20}\left(\varsigma^{2}-\frac{1}{2} r^{2}\right)+a_{30}\left(\varsigma^{3}-\frac{3}{2} \varsigma r^{2}\right)$, $\frac{\partial U}{\partial \varsigma}=a_{10}+2 a_{20} \varsigma+3 a_{30}\left(\varsigma^{2}-\frac{1}{2} r^{2}\right)$,

$\frac{\partial U}{\partial r}=-a_{20} r-a_{30} \varsigma r$

where $\varsigma$ lies in the interval bounded by $z_{i}$ and $z_{i+1}\left(z_{1}\right.$ and $z_{2}$ in Fig.5), and the coefficients can be defined as:

$a_{00}=\frac{1}{h\left(2 R_{0}+h\right)}\left[\left(R_{0}+h\right)^{2} U_{1}-R_{0}^{2} U_{4}\right]$,

$a_{10}=\frac{1}{3 h^{2}\left(2 R_{0}+h\right)}\left\{-\left[3\left(R_{0}+h\right)^{2}+4 h^{2}\right] U_{1}+\right.$

$+\left[3\left(R_{0}+h\right)^{2}-2 h^{2}\right] U_{2}+\left(2 h^{2}-3 R_{0}^{2}\right) U_{3}+$

$\left.+\left(4 h^{2}+3 R_{0}^{2}\right) U_{4}\right\}$

$a_{20}=\frac{2}{h\left(2 R_{0}+h\right)}\left(U_{1}-U_{4}\right)$,

$a_{30}=\frac{2}{3 h^{2}\left(2 R_{0}+h\right)}\left(U_{2}-U_{1}+U_{4}-U_{3}\right)$.

Special care is needed for the electron trajectories which are close to the axis of symmetry where $r \rightarrow 0$ and the radial component of the electrostatic field $E_{r}=$ 0 . Then the system of equations (7) can be written as:

$$
\begin{array}{ll}
\frac{d^{2} z}{d t^{2}}=\frac{e}{m} E_{z 0}, & z(0)=z_{0}, \quad d z /\left.d t\right|_{t=0}=V_{z 0}, \\
\frac{d^{2} r}{d t^{2}}=\frac{r_{0}^{2} V_{\varphi_{0}}}{r^{3}}, & r(0)=r_{0}, \quad d r /\left.d t\right|_{t=0}=V_{r 0}, \\
\frac{d \varphi}{d t}=\frac{r_{0}}{r^{2}} V_{\varphi_{0}}, &
\end{array}
$$

where $E_{z_{0}}$ is the initial axial component of the strength of the electrostatic field, $V_{r 0}$ and $V_{z 0}$ are the initial radial and axial components of the electron velocity respectively. .

The solution of the system of equations (10) is ([21]):

$z=z_{0}+V_{z 0} t-\eta E_{z 0} t^{2} / 2$,
$r=\sqrt{\left(V_{\varphi 0}^{2}+V_{r 0}^{2}\right) t^{2}+2 r_{0} V_{r 0} t+r_{0}^{2}}$

b). Calculations of the electrons emitted from a photocathode require a higher degree of accuracy, and the interpolating polynomial is expressed via potentials at twenty five mesh points (Fig.6). It has been used to calculate the meridional trajectories $\left(V_{\varphi 0}=0\right)$ of the electron beams. Calculations of the radial and axial components of the strength of the electrostatic field are based on Stirling's difference formula [21]. The potential at any point between the mesh nodes is found using the formula:

$U_{k}=U_{j}=U_{i, k}+s\left(f_{i+1 / 2, k}^{1}+f_{i-1 / 2, k}^{1}\right) / 2+s^{2} f_{i, k}^{2}+$
$+s\left(s^{2}-h^{2}\right)\left(f_{i+1 / 2, k}^{3}+f_{i-1 / 2, k}^{3}\right) / 2+s^{2}\left(s^{2}-h^{2}\right) f_{i, k}^{4}$,

where $f_{m, n}^{1} ; f_{m, n}^{2} ; f_{m, n}^{3} ; f_{m, n}^{4}$ are central differences of the first, second, third and fourth order respectively:

$f_{i-3 / 2, k}^{1}=\left(U_{i-1, k}-U_{i-2, k}\right) / h$,

$f_{i-1 / 2, k}^{1}=\left(U_{i, k}-U_{i-1, k}\right) / h$,

$f_{i+1 / 2, k}^{1}=\left(U_{i+1, k}-U_{i, k}\right) / h$,

$f_{i+3 / 2, k}^{1}=\left(U_{i+2, k}-U_{i+1, k}\right) / h$,

$f_{i-1, k}^{2}=\left(f_{i-1 / 2, k}^{1}-f_{i-3 / 2, k}^{1}\right) /(2 h)$,

$f_{i, k}^{2}=\left(f_{i+1 / 2, k}^{1}-f_{i-1 / 2, k}^{1}\right) /(2 h)$,

$f_{i+1, k}^{2}=\left(f_{i+3 / 2, k}^{1}-f_{i+1 / 2, k}^{1}\right) /(2 h)$,

$f_{i-1 / 2, k}^{3}=\left(f_{i, k}^{2}-f_{i-1, k}^{2}\right) /(3 h)$,

$f_{i+1 / 2, k}^{3}=\left(f_{i+1, k}^{2}-f_{i, k}^{2}\right) /(3 h)$,

$f_{i, k}^{4}=\left(f_{i+1 / 2, k}^{3}-f_{i-1 / 2, k}^{3}\right) /(4 h)$.

The potential $U_{m}$ at some point $m$ (Fig.6) can be defined as: 


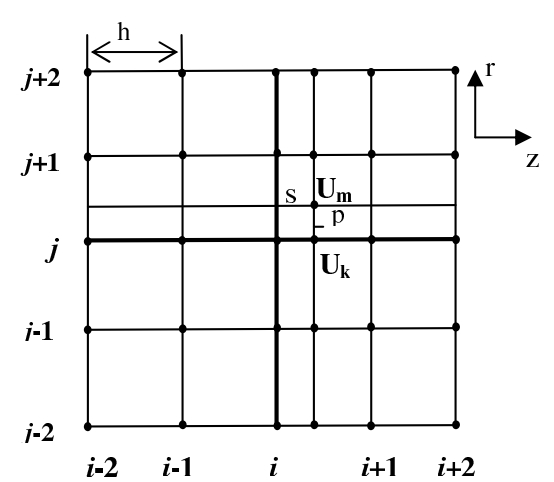

Fig. 6 The grid for calculating the strength of the electrostatic field in EOS

$U_{m}=U_{j}+p\left(f_{j+1 / 2}^{1}+f_{j-1 / 2}^{1}\right) / 2+p^{2} f_{j}^{2}+$
$+p\left(p^{2}-h^{2}\right)\left(f_{j+1 / 2}^{3}+f_{j-1 / 2}^{3}\right) / 2+p^{2}\left(p^{2}-h^{2}\right) f_{j}^{4}$

where the central differences are found using formulae similar to (13).

Differentiating with respect to $p$ we obtain the radial component of the strength of the electrostatic field at the point $m$ :

$$
\begin{aligned}
& -E_{r}=\left(f_{j+1 / 2}^{1}+f_{j-1 / 2}^{1}\right) / 2+2 p f_{j}^{2}+ \\
& +\left(3 p^{2}-h^{2}\right)\left(f_{j+1 / 2}^{3}+f_{j-1 / 2}^{3}\right) / 2+2 p\left(2 p^{2}-h^{2}\right) f_{j}^{4}
\end{aligned}
$$

The axial component of the strength of the electrostatic field can be found using the similar approach, and is defined by the formula:

$$
\begin{aligned}
& E_{z}=\left(f_{i+1 / 2, k}^{1}+f_{i-1 / 2, k}^{1}\right) / 2+2 s f^{2}+ \\
& +\left(3 s^{2}-h^{2}\right)\left(f_{i+1 / 2, k}^{3}+f_{i-1 / 2, k}^{3}\right) / 2+2\left(2 s^{3}-s h^{2}\right) f_{i, k}^{4} .
\end{aligned}
$$

Figure 3 shows central meridional trajectories of the electron beams emitted from the photocathode. The trajectories have been calculated using the algorithm described here.

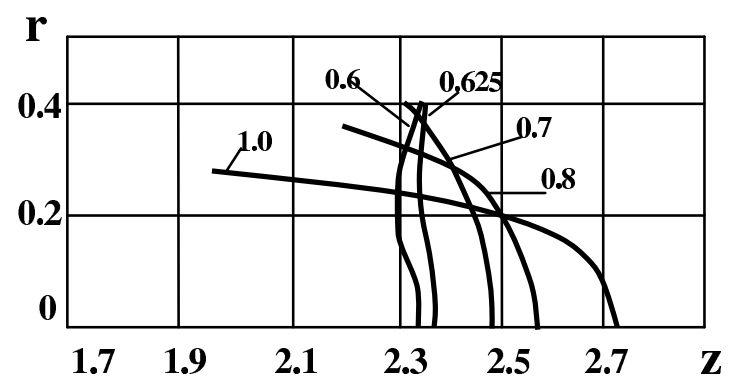

Fig. 7 The curvature of the image surfaces for different radii of the photocathode

\section{Computational Results of EOS}

The major problems which arise in the process of the development of the EOS can be identified as follows:

- the image surface is not flat,

- the quality of the image is nonuniform along the screen, - the electron magnification along the image surface is nonuniform .

To provide high image quality, numerical experiments based on the algorithms described in Sections 2 and 3 have been conducted. The curvature of the image surface has been calculated as a total combination of minimal cross sections of the electron beams (cross-over positions), emitted from different points of the photocathode with the initial energy $\varepsilon_{0}$ and the initial angle $-\frac{\pi}{2} \leq \theta_{0} \leq \frac{\pi}{2}$.

Figure 3 shows the central trajectories of some electron beams where coordinates of the minimal cross sections show the curvature of the image surface. It is seen that the image surface is not flat, and does not coincide with the channel plate. It leads to the nonuniform quality of the image on the screen.

To improve the image quality, the numerical experiments have been conducted for different radii of the spherical photocathode and distances from the photocathode to the anode. The numerical experiments show (Fig.7) that the radius of the photocathode affects the curvature and the position of the image surface. It is seen that the relative radius of the photocathode, $R=0.625$, provides the nearly flat image surface, and determines the position of the MCP in the device.

The nonuniform electron magnification along the image surface causes the image aberration called distortion. The distortion $D$ is calculated as $D=\frac{G-G_{0}}{G_{0}} \times 100$, where $G_{0}$ and $G$ are coefficients of the electron magnification at the centre and other points on the image 


\section{$\mathrm{D}, \%$}

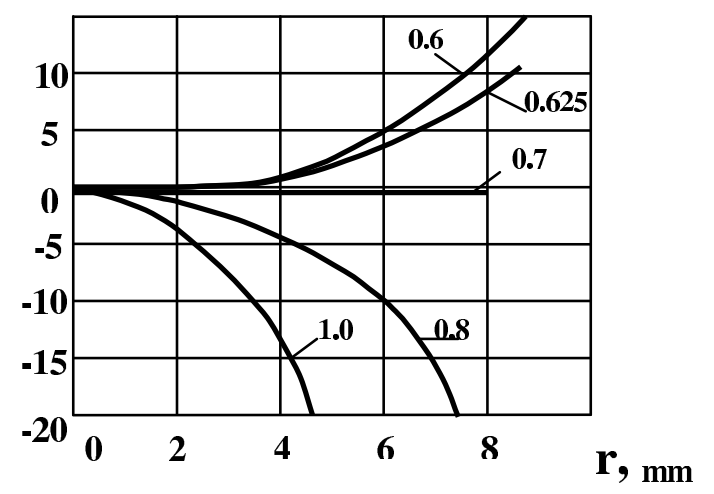

Fig. 8 The distortion of the image for different radii of the photocathode

surface respectively. $D<0$ corresponds to the barrelshaped distortion, and $D>0$ is the case of the pincushion distortion.

The numerical experiments show that the electron magnification also depends on the radius of the photocathode. Figure 8 demonstrates the distortion along the image surface for the different radii of the photocathode. It is seen that the system with relative radius $R=0.7$ is practically without the distortion with $G=1$ along the the image surface. The relative radius $R=0.625$ provides flat image surface with $G_{0}=0.95$ and $G \leq 1.05$ what is relevant to $D \leq 10 \%$.

The numerical experiments, as shown in Figure 9, demonstrate that the distance between the photocathode and anode $\left(L_{c}\right)$ affects the position of the image surface and practically does not affect its shape. Precise determination of the image position defines the position of the channel plate and the length of the device.

\section{Modulation Transfer Function of the Image System}

The usual method of describing the resolution over the image area of an image device is by the modulation transfer function [1], [3], [4]. The MTF gives the dependence of the output contrast on the spatial frequency. As it gives an objective estimation of the image quality, it is used to approximate the position of best focus of an imaging system [8]. The MTF of the overall system, at a given spatial frequency, is the product of the MTFs of the elements. Consequently, to evaluate the total MTF of the imaging system with a micro-channel plate, the

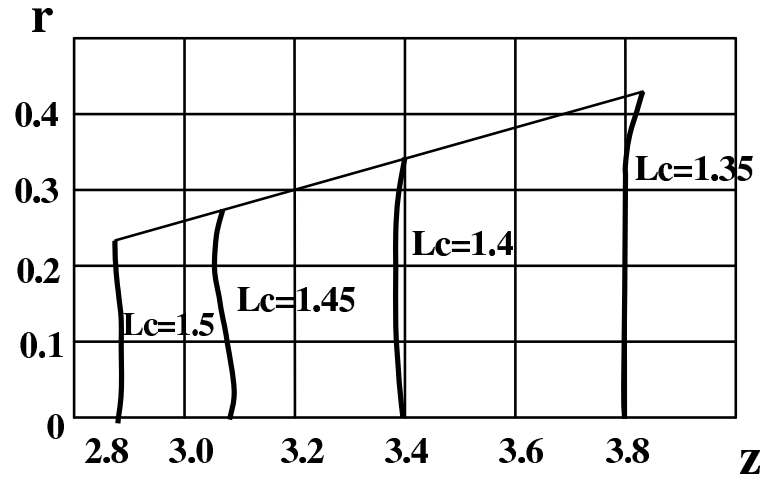

Fig. 9 The image surfaces for different distances from the photocathode to the anode

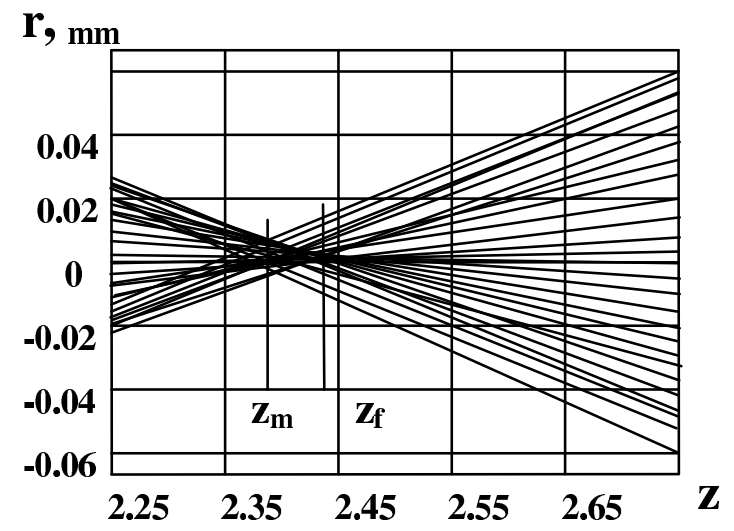

Fig. 10 The trajectories of the electron beam near the screen

MTFs of the electron-optical system and MCP-screen system should be determined.

\subsection{MTF of the electron-optical system}

Figure 10 represents the meridional trajectories of the electron beam near the screen calculated as described in Section 3. It is seen that the image contrast at the point of the best focus $z_{f}$ will be higher than the image contrast at the cross-over position $z_{m}$.

To evaluate the image contrast and precisely define the position of the surface of the best focus in the device, the MTF in the image plane for the point on the axis of the symmetry is calculated using the formula [12]: 


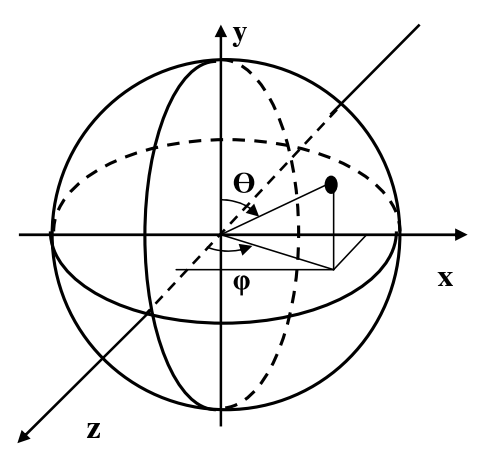

Fig. 11 Emission angles of photoelectrons

$T=\frac{\int_{0}^{\infty} A(r) J_{0}(2 \pi \nu r) r d r}{\int_{0}^{\infty} A(r) r d r}$,

where $J_{0}$ is Bessel's function, $\nu$ is a spatial frequency, and $A(r)$ is an electron density distribution in the cross section of the axial electron beam on the image surface.

To find the electron density function $A(r)$ let assume [2] that $d N(\varepsilon, \theta, \varphi)$ electrons are emitted from the axial point of the photocathode in a time unit with initial energies in the interval $[\varepsilon, \varepsilon+d \varepsilon]$, initial angles $[\theta, \theta+$ $d \theta]$ and $[\varphi, \varphi+d \varphi]$, where $\theta$ and $\varphi$ are meridional and asimuthal angles respectively (Fig. 11).

The number of electrons $d N(\varepsilon, \theta, \varphi)$ depends on the total number of the electrons $N_{0}$ emitted from one point of the photocathode and the probability of the emission $P(\varepsilon, \theta, \varphi)$. Therefore, it can be written:

$d N(\varepsilon, \theta, \varphi)=N_{0} d P(\varepsilon, \theta, \varphi)=N_{0} p(\varepsilon, \theta, \varphi) d \varepsilon d \Omega$,

where $p(\varepsilon, \theta, \varphi)$ is the probability density, and $d \Omega=$ $\sin \theta d \theta$.

Since $\varepsilon, \theta$, and $\varphi$ are independent random variables then $p(\varepsilon, \theta, \varphi)=p(\varepsilon) p(\theta) p(\varphi)$, where $p(\varepsilon), p(\theta)$, and $p(\varphi)$ are probability density functions of the electron's energy, meridional and azimuthal angles respectively. Assuming that $p(\varphi)=2 \pi$ is the uniform distribution the expression for the number of the emitted electrons $d N(\varepsilon, \theta, \varphi)$ can be written as:

$$
\begin{aligned}
& d N(\varepsilon, \theta, \varphi)=N_{0} p(\varepsilon) d \varepsilon p(\theta) \sin \theta d \theta \int_{0}^{2 \pi} p(\varphi) d \varphi= \\
& =N_{0} p(\varepsilon) p(\theta) \sin \theta d \theta d \varepsilon
\end{aligned}
$$

The photoelectrons with the initial energy $\varepsilon$ would have a distance $r$ from the axis of the symmetry at the image surface if their initial angle $\theta$ satisfies a condition $r-f(\varepsilon, \theta)=0$. Using the $\delta$-function the expression for the electron density at the distance $r$ from the axis of the symmetry can be written as:

$$
\begin{aligned}
& d n(r)=\frac{d N(\varepsilon, r)}{2 \pi r}= \\
& =\frac{N_{0} p(\varepsilon)}{2 \pi r} \int_{0}^{\pi / 2} p(\theta) \sin \theta \delta[r-f(\varepsilon, \theta)] d \theta d \varepsilon .
\end{aligned}
$$

Integrating (20) with respect to energy $\varepsilon$ the electron density function $A(r)$ can be expressed as

$$
A(r)=\int_{0}^{\varepsilon_{0}} \frac{N_{0} p(\varepsilon)}{2 \pi r} \int_{0}^{\pi / 2} p(\theta) \sin \theta \delta[r-f(\varepsilon, \theta)] d \theta d \varepsilon .
$$

Substituting (21) to (17) the expression for the modulation transfer functions can be written as:

$$
\begin{aligned}
& T(\nu)= \\
& =\frac{\int_{0}^{\infty} \int_{0}^{\varepsilon_{0}} p(\varepsilon)\left\{\int_{0}^{\pi / 2} p(\theta) \sin \theta \delta[r-f(\theta, \varepsilon)] d \theta\right\} d \varepsilon J_{0}(2 \pi \nu r) d r}{\int_{0}^{\infty} \int_{0}^{\varepsilon_{0}} p(\varepsilon)\left\{\int_{0}^{\pi / 2} p(\theta) \sin \theta \delta[r-f(\theta, \varepsilon)] d \theta\right\} d \varepsilon d r} .
\end{aligned}
$$

Using the property of the $\delta$-function that $\int_{0}^{\infty} \varphi(x) \delta(x-$ $\left.x_{0}\right) d x=\varphi\left(x_{0}\right)$ the final formula for the modulation transfer functions can be obtained:

$T(\nu)=\frac{\int_{0}^{\varepsilon_{0}} p(\varepsilon) \int_{0}^{\pi / 2} p(\theta) \sin \theta J_{0}[2 \pi \nu f(\theta, \varepsilon)] d \theta d \varepsilon}{\int_{0}^{\varepsilon_{0}} p(\varepsilon) \int_{0}^{\pi / 2} p(\theta) \sin \theta d \theta d \varepsilon}$,

where $p(\theta), p(\varepsilon)$ are angular and energy distributions of the electrons respectively (here the angular distribution is described by Lambert's law, $p(\theta)=\cos (\theta)$, and the electron energy is chosen from the parabolic function $\left.p(\varepsilon)=\frac{6 \varepsilon}{\varepsilon_{0}^{2}}\left(1-\frac{\varepsilon}{\varepsilon_{0}}\right)\right) ; \delta$ is a delta function, $\varepsilon_{0}$ is the maximal possible initial energy of the photoelectron; $r=f(\varepsilon, \theta)$ is the distribution of the electron radial coordinates at the image surface which can be written as $([20])$ :

$r(z)=2 \sqrt{\frac{\varepsilon}{E_{0}}} \sin \theta u_{1}(z)-\frac{2 \varepsilon}{E_{0}} \sin \theta \cos \theta u_{2}(z)$,

where $E_{0}$ is the strength of the electrostatic field near the photocathode; $u_{1}(z)$ and $u_{2}(z)$ are two particular solutions of the equation of the motion of paraxial electrons. $u_{1}(z)$ and $u_{2}(z)$ are calculated using the RungeKutta methods as described in the Section 3. 


\subsection{MTF of the MCP-screen}

The use of MCP as the amplifier in the device has many advantages, but the discrete structure of MCP and defocusing of the electron beam in the space between the MCP and the screen, restricts the spatial frequency what can be transferred by the device. A meaningful MTF can be obtained up to the array frequency [3].

The loss in contrast due to the divergence of the electrons in the MCP-screen gap can be reduced by carefully designing the electron lens at the output of each channel. The lens is formed by the penetration of the gap field into a channel [5], [6], and [17]. The limit is set by the smallest gap that will reliably hold of the voltage necessary to excite the phosphor of the screen.

A computer model of the amplification process and the formation of an image of an individual channel in the MCP-screen system has been developed and implemented . The multiplication process of the electron flux in a channel was modeled using Monte-Carlo methods [16]. The inhomogeneous field at the output of the channel (Fig. 4) and the electron trajectories in an inhomogeneous field have been calculated as described in Sections 2 and 3.

Formula (17) is used in computations of the MTF of the MCP-screen system. The electron density distribution $A(r)$ in the image of an individual channel is determined from the arrival coordinates of the electrons at the screen. Taking into account the discret nature of the determination of $A(r)$ here, and using the relationship $\int x^{n} J_{n-1}(x) d x=x^{n} J_{n}(x)$, the calculating formula for MTF can be written:

$T=\frac{\sum_{i=1}^{n} A_{i}\left(r_{i}\right)\left[r_{i+1} J_{1}\left(2 \pi \nu r_{i+1}\right)-r_{i} J_{1}\left(2 \pi \nu r_{i}\right)\right.}{\pi \nu \sum_{i=1}^{n} A_{i}\left(r_{i}\right)\left(r_{i+1}{ }^{2}-r_{i}^{2}\right)}$.

\section{Computational Results of MTFs}

Figure 12 demonstrates the calculated results of the EOS's modulation transfer functions for different initial energies of the electrons emitted from the photocathode. It shows that the electrons with the lower initial energy are better focused by the electrostatic lens which increases the resolution of the device. The initial energy of the photo-electrons depends on the frequency of the incident light and the type of the photocathode used in the device.

Figure 13 shows calculated results of the dependence of the EOS's modulation transfer function on the potential $U_{a}$, applied between the cathode and the anode for $\varepsilon_{0}=0.5 \mathrm{eV}$. It is seen that the developed EOS provides high resolution $\nu \geq 100 \mathrm{~mm}^{-1}$ for the $10 \%$ contrast for

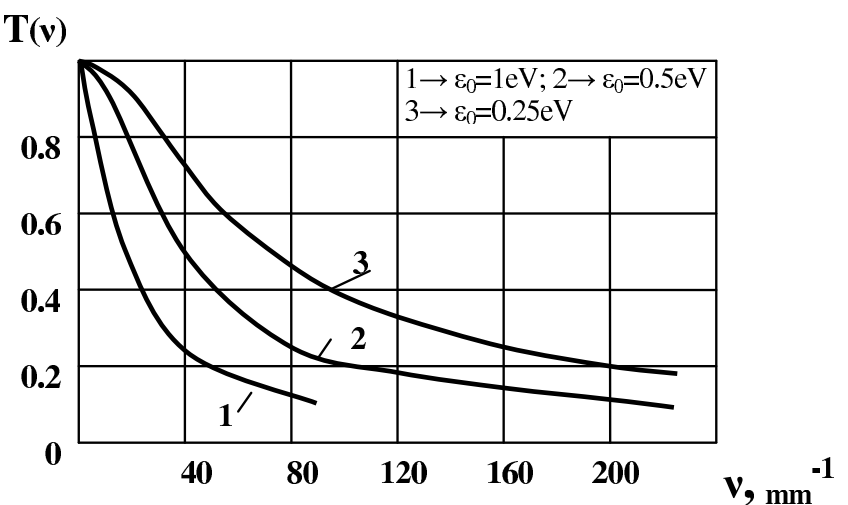

Fig. 12 MTFs of the EOS for the different initial energies of the photoelectrons

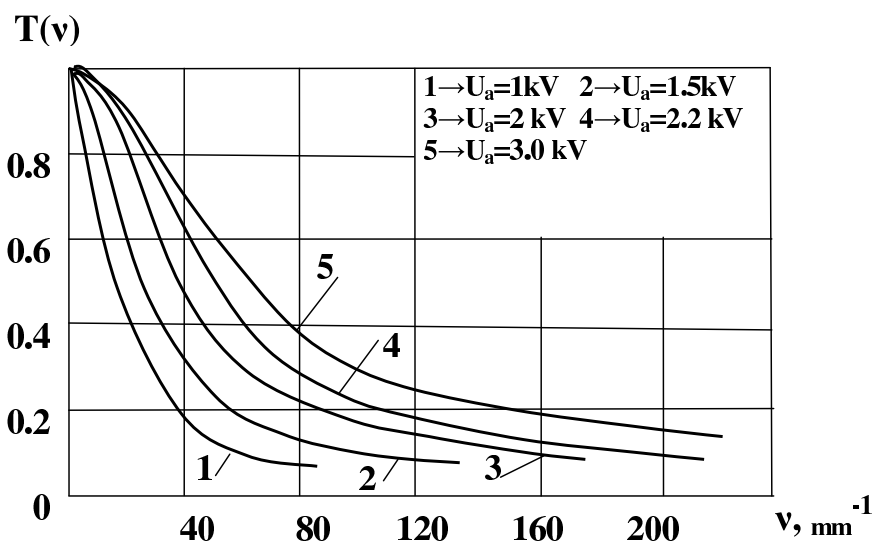

Fig. 13 MTFs of the EOS for the different voltage between the cathode and the anode

quite low applied voltage $\left(U_{a}=1.5-2 \mathrm{kV}\right)$. Such resolution enables the human eye to resolve details of an object in a low level of illumination. The low applied voltage minimizes a noise factor of the MCP [16] which affects the visual acuity of an imaging device.

Figure 14 displays the MTFs of the MCP-screen system, where the solid curves represent calculations and dashed curve represents experimental results [5]. Computational and experimental results were obtained for the channel diameter $d=10 \mu \mathrm{m}$, the voltage on the channel $U_{c}=800 \mathrm{~V}$, the sputtering depth of the contact layer at the channel output $h=1.5 d$, the strength of the electrostatic field $E=5 \mathrm{kV} / \mathrm{mm}$. The distance between MCP and the screen $D=1 \mathrm{~mm}$ for the curves (2) and (3), and $D=0.4 \mathrm{~mm}$ for the curve (1). 


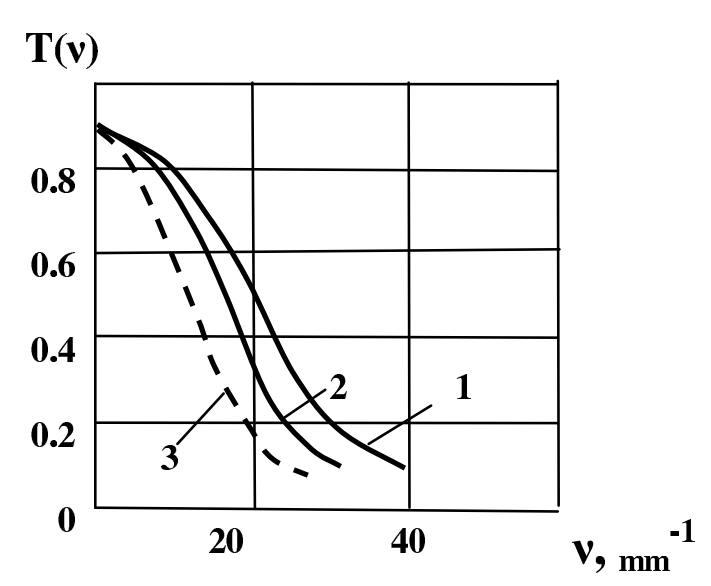

Fig. 14 MTF of the MCP-screen (the solid curves represent calculations and dashed curve represents experiment)

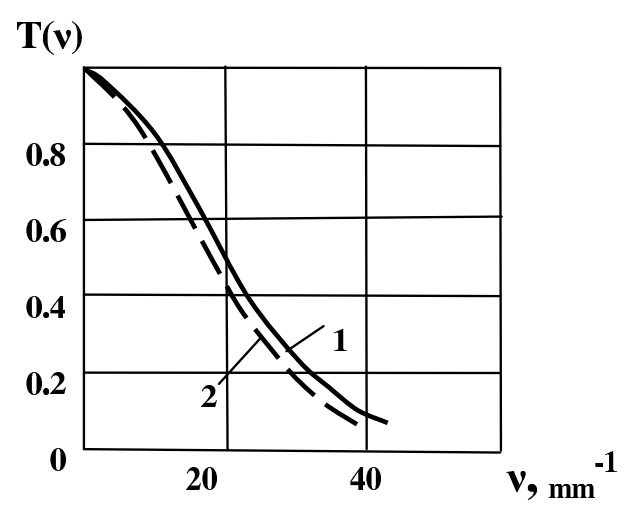

Fig. 15 Final MTF of the imaging system (the solid curve represents calculations and dashed curve represents experiment)

The final MTF of the image system is the product of the MTFs of the EOS (Fig. 13) and MCP (Fig. 14). Figure 15 shows the total MTF of such imaging system where the solid curve represents calculations $(d=10 \mu m)$ and dashed curve represents experiment $(d=12 \mu \mathrm{m}[4])$ for the MCP-screen gap $D=0.4 \mathrm{~mm}$.

\section{Conclusions}

A computational algorithm for calculation of an inverting electron optical system with microchannel amplifiers has been developed. It is consists of:

(a) calculation of the potential distribution inside the imaging systems with rotational symmetry;

(b) calculation of the electron trajectories in the electrostatic field;

(c) determination of the modulation-transfer-function which defines the resolution of the imaging system.
The effect of the system parameters on the image quality has been investigated. Dependance of the curvature of the image surface and image distortion on the radius of the photocathode and the distance between the photocathode and anode have been calculated.

The modulation-transfer-functions of the EOS for the different initial energies of the photoelectrons have been calculated. The dependence of the MTFs of the EOS on the voltage between the photocathode and anode have been investigated. The modulation-transferfunction of the microchannel amplifier - screen with an inhomogeneous field at the output of the channel has been calculated, and the total MTF of the imagine system has been defined.

The electron optical system, developed in this work, has practically uniform quality of the image on the screen, low distortion and high resolution. The flat image surface provides a uniform image quality, and therefore allows the use of a micro-channel plate as an amplifier. The low applied voltage minimizes a noise factor of the MCP. All dimensions and parameters of the imaging system have been defined precisely and left outside this publication.

ACKNOWLEDGEMENT. The author wishes to acknowledge the contribution made to this work by V.N. Evdokimov.

\section{References}

1. Catchpole, C.E.: Measurement of the spatial frequency response of image devices. Advan. Electron. Electron. Phys. 22A, 58, 425-433 (1966)

2. Ciganenko, V.V. and Choporov, P.P.: Electronic Technology 4, $3(1970)$

3. Clarke, J.A.: Measuring the MTF of channel image intensifiers. Acta Electronica 16, 1, 33-41 (1973)

4. Emberson, D.L., Holmshaw, R.T.: The design and performance of an inverting channel image intensifier. Acta Electronica 16, 1, 23-32 (1973)

5. Evdokimov, V.N., Kudrya, A.A., Tyutikov, A.M., Flegontov, Yu.A., Shymanska, A.V.: Current density distribution in the image of the multidyne's channel. Radiotekh. Electron. 29, 390-392 (1984)

6. Hoenderken, T. H., Hagen, C. W., Barth, J. E., Kruit, P., Nutzel, G.O.: Influence of the microchannel plate and anode gap parameters on the spatial resolution of an image intensifier. J.Vac.Sci.Technol. B19(3), 843-850 (2001)

7. Ilyin, V.P.: Numerical methods for solving problems in electron optics. Nauka Press, Novosibirsk(1974)

8. Ivanov, V.: The image quality evaluation. Microchannel amplifiers. The University of Chicago, Argonne and Fermilab, Large-area picosecond photo-detectors project, USA (2009). http : //psec.uchicago.edu/Papers/Ivanov_image_quality.doc

9. Ivanov, V., Brezhnev, V.: New formulation of the synthesis problem in electron optics. Nucl.Instrum.Methods A519, 117132 (2004) 
10. Ivanov, V., Kriklivyy, V.: Numerical algorithms for boundary problems with disturbed axial symmetry. Nucl.Instrum.Methods A519, 96-116 (2004)

11. Khursheed, A.: The finite element method in charged particle optics. Kluwer Academic Publishers, Boston/Dordrecht/London,1-60(1999)

12. Miroshnikov, M.M.: Theoretical foundations of electron - optical devices. Mechanical Engineering, Leningrad (1983)

13. Munro, E.: Computational techniques for design of charged particle optical systems. In: Orloff, J. (edt.) Handbook of charged Particle Optics,pp. 1-74. CRC Press LLC, (1997)

14. Schagen, P.: Electronic aids to night vision. Philos. Trans. R. Soc. A269, 1196, 233-263 (1971)

15. Schagen, P.: Image converters and intensifiers. J. Phys. E.: Sci.Instr. 8, 153-160 (1975)

16. Shymanska, A.V.: Computational modeling of stochastic processes in electron amplifiers. J. Comput. Electron. 9, 93-102 (2010)

17. Shymanska, A. V., Evdokimov, V. N.: Effect of parameters of multidyne screen system on image quality. Sov. J. Opt. Technol. 52(7), 393-394 (1985)

18. Siegmund, O., Vallerga, J.V., Tremsin, A.S., Feller, W.B.: High Spatial and Temporal Resolution Neutron Imaging With Microchannel Plate Detectors. IEEE Transactions on Nuclear Science. 56(3), 1203 - 1209 (2009)

19. Tremsin, A. S., Feller, W.B., Dowing, R.G.: Efficiency optimization of microchannel plate neutron imaging detectors. Nucl.Instrum.Meth. A539(1-2), 278-311 (2005)

20. Vorobyov, Yu. V.: Journal Technical Physics 26, 2269 (1956)

21. Weisstein, E. W.: CRC Concise encyclopedia of mathematics, CRC Press, Boca Raton, 1728, 1740 (1998)

22. Wiza, J.L.: Microchannel plate detectors. Nucl.Instr.Methods. A 162, 587-601 (1979)

23. Young, D.M.: Iterative solution of large linear systems, Dover, New York(2003) 